\title{
EL FENÓMENO URBANO Y LA SITUACIÓN EN LOS PAÍSES LATINOAMERICANOS. EMPLAZAMIENTOS URBANOS EN ZONAS DE RIESGOS NATURALES. EL CASO DEL GRAN RESISTENCIA.
}

\author{
THE URBAN PHENOMENON AND THE SITUATION IN LATIN AMERICAN COUNTRIES. URBAN \\ SITES IN NATURAL HAZARD AREAS. THE CASE OF "EL GRAN RESISTENCIA."
}

\author{
Viviana Claudia Pertile \\ vpertile@hum.unne.edu.ar \\ Instituto de Geografía- UNNE
}

\begin{abstract}
Resumen
El fenómeno urbano del siglo XX se ha caracterizado por el crecimiento explosivo de la urbanización en el mundo, como así también por la complejidad que han adquirido las formas y funciones urbanas. El área Metropolitana del Gran Resistencia constituye un núcleo regional de gran importancia tanto desde el punto de vista de la población como de las funciones que desempeña en el nordeste argentino. Al mismo tiempo debemos decir que la ciudad se localiza en una planicie aluvional dominada por la acción de cursos de agua, a lo que se le suma un régimen de lluvias abundantes, con un doble máximo, uno en noviembre y otro en marzo. Estos hechos determinan que la ciudad se encuentre bajo la amenaza de sufrir anegamientos periódicos, situación que se agrava por la falta de regulaciones urbanísticas que orienten el crecimiento del área metropolitana.
\end{abstract}

\section{Palabras Clave:}

Fenómeno urbano - vulnerabilidad física - mitigación.

\begin{abstract}
The urban phenomenon of the twentieth century has been characterized by the explosive growth of urbanization in the world, as well as by the complexity of urban forms and functions. The Metropolitan Area of El Gran Resistencia is a regional center of great importance not only from the viewpoint of the population but also for the functions it performs in the northeast of Argentina. The city is located on an alluvial plain dominated by the action of water courses, to which is added an abundant rainfall regime, with a double maximum, one in November and another in March. These facts determine that the city is under the threat of having periodical flooding, a situation aggravated by the lack of urban regulations to guide the growth of the metropolitan area.
\end{abstract}

Keywords:

Urban phenomenon - physical vulnerability - mitigation.

\section{Presentación}

El presente Ensayo tiene por objeto analizar el Proceso de Urbanización en los Países Subdesarrollados y los problemas que ello ocasiona en los Emplazamientos Urbanos en Zonas de Riesgos Naturales. El caso de Resistencia

Para lograr este fin hemos dividido nuestro trabajo en tres secciones. La primera se dedica a exponer algunos ejes conceptuales del proceso de urbanización. En la segunda sección se analizan los problemas que ocasionan las altas concentraciones de población de en ciudades con riesgos naturales y

Publicado en formato digital: Mgter. Viviana Claudia Pertile. EL FENÓMENO URBANO Y LA SITUACIÓN EN LOS PAÍSES LATINOAMERICANOS. EMPLAZAMIENTOS URBANOS EN ZONAS DE RIESGOS NATURALES. EL CASO DEL GRAN RESISTENCIA.). Revista Geográfica Digital. IGUNNE. Facultad de Humanidades. UNNE. Año 9. № 18. Julio - Diciembre 2012. ISSN 1668-5180 Resistencia, Chaco. En: http://hum.unne.edu.ar/revistas/geoweb/default.htm 
Revista Geográfica Digital. IGUNNE. Facultad de Humanidades. UNNE. Año 9. № 18. Julio - Diciembre 2012. ISSN 1668-5180 Resistencia, Chaco

en la tercera se esboza, a modo de propuesta, una perspectiva superadora que aborda de manera integral las posibilidades de intervención en la problemática planteada.

\section{Primera Sección \\ Ejes conceptuales del proceso de urbanización}

Mariano Zamorano (1977) plantea el fenómeno urbano del siglo XX como una crisis de un proceso, sostienen que el crecimiento explosivo de la urbanización en el mundo, particularmente en los últimos cuarenta años y acompañado por la complejidad que han adquirido las formas y funciones urbanas, han desdibujado de manera notable el concepto de ciudad. Si el sentido clásico de este término nos colocaba frente a un modo de poblamiento bien discernible, en nuestros días cuesta establecer con claridad su estilo representativo y el papel que le corresponde. Por eso se prefiere utilizar la expresión FENÓMENO URBANO, en alusión al carácter rápido, desmesurado y detonante de la formidable expansión de este proceso de desarrollo espacial y de concentración de población.

Hasta comienzos de este siglo, existía una oposición nítida entre el campo y la ciudad; en principio mayor tamaño, trazado y arquitectura propia, superior dinamismo y actividades variadas, fundamentalmente no agrícolas.

En la segunda mitad de este siglo, en cambio, nuevas fórmulas surgieron: conjuntos poblacionales periféricos para miles de personas, agrociudades nacidas con el impulso colonizador, ciudades obreras transitorias por esencia al servicio de una explotación minera o de emprendimientos hidroeléctricos, complejos turísticos con una gran infraestructura comercial y de esparcimientos, pueblos transformados prácticamente en dormitorios para quienes trabajan en la urbe, etc.

La ciudad no es un hecho reciente; cada época histórica, a partir del Neolítico, tuvo sus hechos urbanos que hasta hoy la representan. Sin embargo en el siglo XIX se produce una transformación muy grande del hábitat, razón por la cual el crecimiento de la ciudad adquiere una dimensión hasta entonces jamás vista, extraordinaria, espectacular, que fue definida como "fenómeno urbano".

Jaqueline Beaujeu Garnier y Georges Chabot (1975), en su Tratado de Geografía Urbana ilustran esta afirmación con los siguientes datos estadísticos (en localidades de más de 5.000 habitantes):

\begin{tabular}{|l|c|c|}
\hline \multicolumn{1}{|c|}{ Períodos } & $\begin{array}{c}\text { Crecim. de la } \\
\text { Poblac. Mundial }\end{array}$ & $\begin{array}{c}\text { Crecim. de la } \\
\text { Poblac. Urbana }\end{array}$ \\
\hline $1800-1850$ & $29,2 \%$ & $175,4 \%$ \\
\hline $1850-1900$ & $37,3 \%$ & $192,0 \%$ \\
\hline $1900-1950$ & $49,2 \%$ & $228,0 \%$ \\
\hline
\end{tabular}

Desde 1950 a la fecha el crecimiento se acentuó aún más, tanto, que algunos geógrafos se permiten anticipar que la civilización del futuro será eminentemente urbana y que aproximadamente el $90 \%$ de la población vivirá en ciudades. La República Argentina cuenta ya con una población urbana de $88 \%$, es decir, habitando en centros de más de 2.000 habitantes. En el Chaco es del $70 \%$. Las razones de este crecimiento desmesurado se deben buscar en la misma humanidad que se ve impulsada a nuevas formas de civilización más urbana y más humana, a su carácter gregario, y a una ocupación cada vez más disociada de los trabajos de la tierra, como lo son la industria, el comercio, la educación, los medios de transporte, la sanidad, etc.

De acuerdo con las proyecciones del Banco Mundial, hacia el 2025 más de 5 mil millones de personas, es decir dos tercios de la población del planeta, vivirá en ciudades; las cifras convocan al vértigo se piensa que hace apenas 50 años el $83 \%$ de la personas vivían en el campo. La inmensa mayoría de esas multitudes vivirá en países pobres, en ciudades con déficit fundamentales, inseguras cuando no insalubres. Se estima que dentro de 11 años, casi mil millones y medio de personas vivirán pueblos sin acceso a al agua potable y desagües sanitarios. Las proyecciones de estas megaciudades

Publicado en formato digital: Mgter. Viviana Claudia Pertile. EL FENÓMENO URBANO Y LA SITUACIÓN EN LOS PAÍSES LATINOAMERICANOS. EMPLAZAMIENTOS URBANOS EN ZONAS DE RIESGOS NATURALES. EL CASO DEL GRAN RESISTENCIA.). Revista Geográfica Digital. IGUNNE. Facultad de Humanidades. UNNE. Año $9 . N^{\circ} 18$. Julio - Diciembre 2012. ISSN 1668-5180 Resistencia, Chaco. En: http://hum.unne.edu.ar/revistas/geoweb/default.htm 
están en estrecha relación con niveles de violencia, desempleo y la propagación de enfermedades contagiosas.

El Secretario de la cumbre de Naciones Unidas, Habitat II, en oportunidad de la Conferencia Mundial sobre asentamientos humanos, celebrada en Estambul en 1996 (Dr. En Cs. Veterinarias Wally N'Down) sostuvo que "estamos pasando de una civilización antes tradicionalmente rural y después nacional a una civilización global,....y estamos siendo testigos de la mayor migración de la historia..., donde las ciudades se están convirtiendo en centros regionales, que pertenecen políticamente a la nación en la que están insertas, pero en términos culturales pertenecen al mundo. (DIARIO CLARÍN, 1996)

\section{La ciudad como fenómeno urbano}

Definir la ciudad actual es una cuestión de paisaje. Sin embargo este aspecto es muy amplio, ya que cada región tiene su forma de paisaje urbano y si comparamos una ciudad con otra advertiremos diferencias. Para definirla se han ensayado diversos criterios, como por ejemplo el numérico, el fisonómico, el funcional, el histórico, etc.

La gran diversidad de criterios y definiciones, señalan con claridad la gran amplitud que tiene el fenómeno urbano: concentración de habitantes, actividad, género de vida, funciones, esto demuestra que cada definición tiene sus límites.

El hecho es complejo y abarca muchas actividades e indica gamas de posibilidades urbanas desde la Megalópolis hasta las ciudades de países poco desarrollados.

CIUDAD: fenómeno importante, permanente, organizado para la vida colectiva y de actividad en un marco espacial determinado (las densidades urbanas son superiores a las rurales). Fenómeno de actividad, porque son actividades no agrícolas, especialmente de tipo secundario y terciario, en un marco determinado.

De modo que definir la ciudad no es tema fácil ya que se trata de un hecho complejo, variable e importante. No obstante, se han intentado aproximaciones para definirla, a partir de ciertos criterios mencionados anteriormente.

La mejor definición de ciudad es aquella que reúne el mayor número de criterios, como por ejemplo un número importante de habitantes, profesiones ligadas a las actividades secundarias y terciarias, con un paisaje compacto y el reconocimiento de su evolución histórica.

Max Derruau (1964), en su Tratado de Geografía Humana define a la ciudad como una aglomeración importante, organizada para la vida colectiva (esta organización es el urbanismo) y con la mayoría de la población dedicada a las tareas no agrícolas.

\section{Segunda Sección}

\section{Algunas características de las ciudades de América Latina}

La mayoría de los países Latinoamericanos están experimentando un ritmo de crecimiento significativo, tanto de sus habitantes como en el número de ciudades, a lo que hay que añadir perspectivas preocupantes por el ritmo a que se ven sometidos estos países, que no cuentan con el soporte básico de una economía que permita la elevación de su nivel de vida o la planificación sistemática para poder controlar dos variables: población y economía (RICA, 1990).

Los problemas más graves que debe afrontar la humanidad en los próximos años, son precisamente los que corresponden a los países subdesarrollados. En la actualidad una de cada dos personas viven en estos países.

Las ciudades de estos países atraen cada día a miles de desempleados y a quienes buscan mejorar su modo de vida.

La ONU afirma que en estos momentos existen 1.200 millones de personas que habitan en condiciones precarias en los grandes núcleos urbanos, ya sea por que no tienen agua, electricidad y vías de acceso o por que carecen de condiciones sanitarias o educativas.

Publicado en formato digital: Mgter. Viviana Claudia Pertile. EL FENÓMENO URBANO Y LA SITUACIÓN EN LOS PAÍSES LATINOAMERICANOS. EMPLAZAMIENTOS URBANOS EN ZONAS DE RIESGOS NATURALES. EL CASO DEL GRAN RESISTENCIA.). Revista Geográfica Digital. IGUNNE. Facultad de Humanidades. UNNE. Año $9 . N^{\circ} 18$. Julio - Diciembre 2012. ISSN 1668-5180 Resistencia, Chaco. En: http://hum.unne.edu.ar/revistas/geoweb/default.htm 
Revista Geográfica Digital. IGUNNE. Facultad de Humanidades. UNNE. Año 9. № 18. Julio - Diciembre 2012. ISSN 1668-5180 Resistencia, Chaco

Si bien las fuentes de información son desiguales como desigual es el grado de urbanización de América Latina, se han obtenido algunas características demográficas comunes para el conjunto de países que la integran: 1.- En las grandes ciudades la tasa de fecundidad es débil. 2.- El índice de masculinidad es menor que en las áreas rurales. 3.- La nupcialidad es mayor (todavía). 4.- La proporción de jóvenes y adultos es elevada. 5.- La tasa de analfabetos es sensiblemente menor. 6.- La tasa de mortalidad general no presenta diferencias, pero la mortalidad infantil es menor en las ciudades. 7.- El rápido crecimiento de las ciudades, en particular de las más grandes se explica esencialmente por las migraciones campo-ciudad. 8.- El crecimiento natural es semejante en áreas urbanas y rurales. 9.- La población rural que se instala en las ciudades es en su mayoría de sexo femenino de entre 15 y 39 años. 10.- De las migraciones internacionales que contribuyeron al crecimiento de las ciudades, predominó el sexo masculino. 11.- El número de activos masculinos y de obreros industriales es semejante a la de los países más evolucionados, el de los servicios, es mayor y en el de comercio, es menor (RICA, Op. Cit).

Desde el punto de vista sociológico, fueron varios los aspectos que interesaron respecto de la urbanización en los países latinoamericanos, que dieron lugar a reflexiones sociológicas sobre temas como la pobreza, la desocupación y los comportamientos sociopolíticos. (Lezama, 1993); el autor sostiene que la discusión más sociológica sobre la relación urbanización y sociedad en América Latina es la que gira alrededor de la teoría de la marginalidad, en la medida en que en ella convergen los paradigmas más representativos de la disciplina, incluyendo un intento de conceptualización respecto de la situación social y material de lo latinoamericano y reflexiones sobre las conductas sociales.

Las ciudades en Latinoamérica, han sido desde sus orígenes, el ámbito donde se produjo el ordenamiento y estructuración de los distintos momentos históricos y de las diversas formas de organización económica, política y social de las sociedades que la integran. Es en la ciudad donde se concretan las diversas relaciones de intercambio rural-rural o rural-urbano; una de las características que adquieren estas ciudades es el desorden y la pobreza, elementos que con mayor frecuencia se le asignan a la marginalidad.

La pobreza y la marginalidad en las sociedades Latinoamericanas, no son en sí los hechos sobresalientes, sino las dimensiones y el hecho de sustentar mecanismos de reproducción, no tan solo de manera transitoria sino de forma estructural.

Los primeros estudios sobre marginalidad en Latinoamérica intentaban explicar las condiciones de vida de los pobladores de las periferias de las ciudades, asentados en terrenos invadidos y en viviendas deterioradas; La segunda connotación de la marginalidad, está presente en algunos de los planteamientos de la teoría del desarrollo y en el enfoque dependentista. El rasgo más sobresaliente de la marginalidad para este enfoque es la proliferación de focos de miseria y zonas periféricas. Designa a aquellos grupos sociales que aún perteneciendo a una sociedad, no penetran en la intimidad de sus estructuras; no gozan de los beneficios de la vida moderna y están incapacitados para poner fin a la situación que viven y cuya condición marginal afecta al conjunto de su vida. Asimismo sostiene que, independientemente de las causas más profundas que explican la marginalidad, percibe que el origen rural de los marginados de las ciudades agrava la misma situación en la que viven los integrantes de dicho sector. Generalmente el migrante rural llega a las ciudades sin capacitación previa, ingresando a los sectores de más bajos niveles de ingreso y productividad o bien no llegan a insertarse en el mercado de trabajo. Esta situación, los lleva a establecerse en aquellos barrios en los que el acceso a la vivienda está al alcance de sus escasos ingresos. Estos lugares son, casi siempre, zonas de asentamientos irregulares, con condiciones higiénicas precarias, con servicios deficientes o nulos y sin acceso a servicios de educación

\section{Nuestro Objeto de Estudio: El Caso del Gran Resistencia}

Podríamos decir que Resistencia es un caso típico de emplazamiento urbano en zonas de riesgos naturales. El definir al conglomerado urbano del Gran Resistencia a partir de sus características físicas resulta de fundamental importancia para considerar el problema que se deriva por la configuración del sitio en el cual se halla emplazado. En efecto, una parte importante del área urbanizada del mismo se encuentra asentada sobre el lecho de inundación del Río Paraná, que representa dos claros aterrazamientos: uno bajo y próximo al río, donde se ubican las localidades portuarias de Barranqueras y

Publicado en formato digital: Mgter. Viviana Claudia Pertile. EL FENÓMENO URBANO Y LA SITUACIÓN EN LOS PAÍSES LATINOAMERICANOS. EMPLAZAMIENTOS URBANOS EN ZONAS DE RIESGOS NATURALES. EL CASO DEL GRAN RESISTENCIA.). Revista Geográfica Digital. IGUNNE. Facultad de Humanidades. UNNE. Año $9 . N^{\circ} 18$. Julio - Diciembre 2012. ISSN 1668-5180 Resistencia, Chaco. En: http://hum.unne.edu.ar/revistas/geoweb/default.htm 
Vilelas y parte de Resistencia, y otra, a cotas superiores, donde se desarrolla el resto de la ciudad y el núcleo de Fontana.

El Área Metropolitana del Gran Resistencia se localiza en una planicie aluvional dominada por la acción de tres cursos de agua: el Río Paraná, el río Negro y el riacho Arazá. Una serie de lagunas semilunares -muchas de ellas hoy colmatadas- que son restos de antiguos meandros del Río Negro, completan el sistema fluvio-lacustre local, directamente regulado por las fluctuaciones del Paraná. Precisamente las marcadas fluctuaciones extra anuales del Paraná, conjuntamente con los desbordes del río Negro, provocan las inundaciones que recurrentemente afectan al área.

En épocas de lluvias, las aguas precipitadas, que normalmente escurren a través de los cauces o en forma laminar, encuentran obstrucciones de diversa naturaleza que se oponen a su desplazamiento, agregándose a este hecho las acciones antrópicas propias del área urbanizada que limitan fuertemente la capacidad de absorción de los excesos de agua. Por otro lado, el río Negro y el riacho Arazá se encuentran en estadío de colmatación extrema debido a la poca pendiente, al escaso caudal y a la insuficiente capacidad de arrastre, hecho que debilita la evacuación natural de estos cursos en tiempos normales. (Popolizio, 1989)
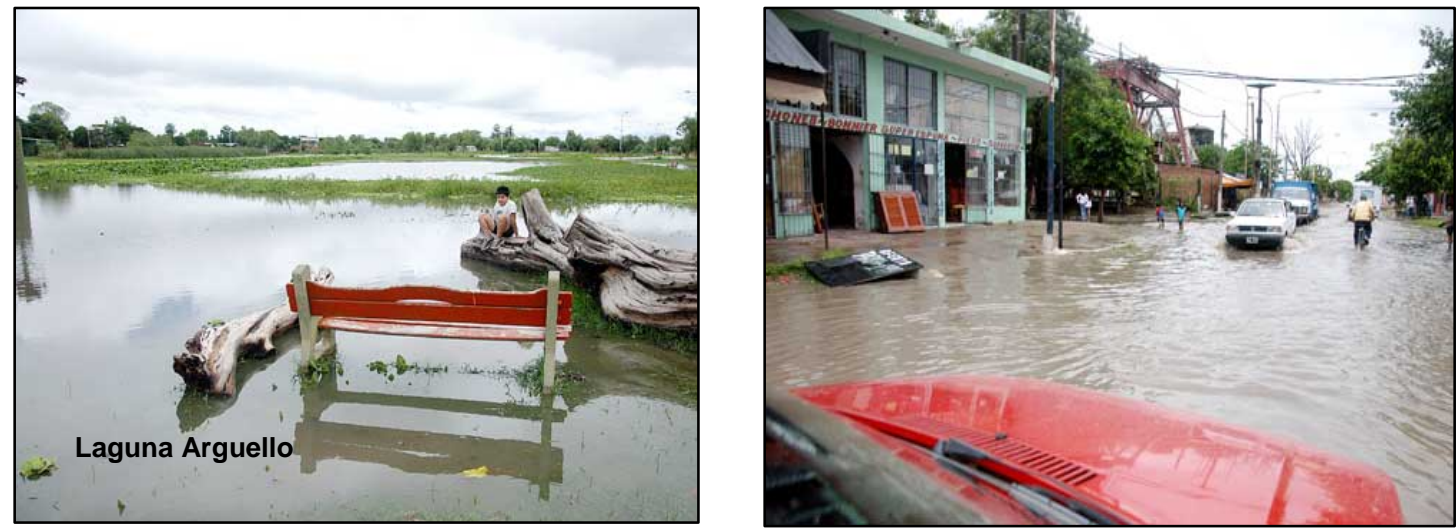

Fuente: El Diario. http:/leldiariodelaregion.org.ar/zenphoto/resistencia-otra-vez-inundada/07-Iluvia.jpg.php

Con estas características del sitio, altamente desfavorable para sostener a una población estable, gran parte del área urbanizada está bajo la amenaza de sufrir anegamientos periódicos, hecho que se agrava por la falta de regulaciones urbanísticas que orienten el crecimiento del área metropolitana, con el consiguiente aumento de la criticidad propia del sitio.

A estas condiciones ambientales-naturales, se suman las modificaciones que el Hombre introdujo en el sistema natural, dando origen a problemas que adquieren gran magnitud a escala social, tanto en el medio local como regional. De este modo, las inundaciones, se instalan en el escenario de la toma de decisiones, por parte de los responsables gubernamentales, encargados de enfrentar y resolver esta problemática. Los años transcurridos bajo las aguas, nos han demostrado que se tomaron caminos equivocados, intentando dominar o manejar la naturaleza como al resto de los sistemas productivos. Es evidente que la ejecución de obras no alcanzó para resolver los problemas. A estas obras hay que mantenerlas, administrarlas y manejarlas y eso no sucedió.

Las inundaciones que han afectado al Gran Resistencia produjeron crisis que repercutieron en todo el ámbito del espacio urbano y determinaron rápidas modificaciones en el mismo, de manera que podríamos incluirla dentro de las denominadas Ciudades en Riesgo, cuya acelerada urbanización y empobrecimiento de grandes grupos poblacionales hicieron que se incrementara el grado de vulnerabilidad ante los desastres naturales. Esta vulnerabilidad es la que Wilches-Chaux (1988) denomina vulnerabilidad física o localizacional, ya que se caracteriza al medio natural, partiendo de la génesis de las inundaciones, destacando los factores globales y locales (los regímenes fluviales, la disposición urbana, las precipitaciones locales) así como otras causas (la deforestación, la corriente del Niño...).

Publicado en formato digital: Mgter. Viviana Claudia Pertile. EL FENÓMENO URBANO Y LA SITUACIÓN EN LOS PAÍSES LATINOAMERICANOS. EMPLAZAMIENTOS URBANOS EN ZONAS DE RIESGOS NATURALES. EL CASO DEL GRAN RESISTENCIA.). Revista Geográfica Digital. IGUNNE. Facultad de Humanidades. UNNE. Año 9. Julio - Diciembre 2012. ISSN 1668-5180 Resistencia, Chaco. En: http://hum.unne.edu.ar/revistas/geoweb/default.htm 
Revista Geográfica Digital. IGUNNE. Facultad de Humanidades. UNNE. Año 9. № 18. Julio - Diciembre 2012. ISSN 1668-5180 Resistencia, Chaco

Las respuestas o soluciones ensayadas, no hicieron más que acelerar y expandir el proceso de degradación del "Sistema Complejo", al decir de Rolando García (1988), en tanto "sistema global" cuya investigación permite estudiar "un trozo de la realidad", que abarca aspectos físicos, biológicos, sociales, económicos y políticos. Las formas de abordar estos sistemas son variadas, siendo lo más difícil definir sus componentes, como así también su organización o estructura y sus límites.

Los sistemas complejos que se presentan en la realidad empírica carecen de límites precisos en su extensión física y en su problemática, de manera que al definirlos hay que reducir al mínimo la arbitrariedad en la partición que se adopte y al hacerlo hay que considerar las interacciones con el medio externo, como así también las condiciones en los límites que se especifican en forma de flujos y dentro de estos, lo más importante es considerar la velocidad de cambio, relacionada con la escala temporal del fenómeno que se estudia. Esto permite investigar la dinámica del sistema y no un estado en un momento determinado. Por lo tanto la característica central de estos sistemas complejos está dada por el conjunto de relaciones entre las diferentes estructuras internas de los mismos.

Esta idea de Sistema Complejo es considerada en el presente trabajo, como fundamental para el análisis central de las inundaciones locales. En los últimos 25 años, los sucesivos gobiernos de la Provincia del Chaco, se fueron olvidando de lo aprendido por el conjunto de la Humanidad [1] y sucumbieron a la tentación de grandes obras de ingeniería o a la inmediatez de atender la emergencia hídrica.

De allí, la necesidad de elaborar proyectos de desarrollo local mediante la asignación de políticas y medidas que tiendan a reducir el riesgo. Al respecto Tyller Miller, G, JR (1992) manifiesta que el análisis del riesgo implica la identificación de los peligros, valoración de la naturaleza y severidad de los riegos (evaluación del riesgo), utilización de esa y otra información para determinar opciones y tomar decisiones acerca de la reducción o eliminación de los riesgos (manejo del riesgo) y comunicación de la información acerca de los riesgos a los decisores o tomadores de decisiones y al público (comunicación del riego).

También es importante considerar aspectos relacionados con la degradación del ambiente, donde la explosión demográfica, se constituye en una de las causas principales de esta degradación (Veyret, I. y Pech, P. (1995), a lo que se agrega la inexistencia de planificación urbana, la falta de políticas de dotación de viviendas en áreas seguras, entre otros. Dar respuestas a estas cuestiones, es muy difícil, por cuanto implica integrar toda una serie de factores dentro de un enfoque sistémico.

Ante estas circunstancias nos preguntamos:

¿Cómo fomentar un desarrollo sostenible teniendo en cuenta la vulnerabilidad que presentan los actores ante los fenómenos y las características del entorno urbano, social y económico que los envuelve?

Para dar respuesta a esta cuestión, proponemos intervenir en torno un eje, que si bien, es fundamental, no es el único posible y necesario de abordar:

\section{Prevención y mitigación de desastres}

En Primer lugar, es esencial que el tema ocupe un lugar de interés en la agenda pública de los poderes y autoridades locales, con el objetivo de emprender acciones para incorporar el factor de evaluación del riesgo en ciudades con alto crecimiento poblacional, cuando lo que se decide tiene que ver con los planes urbanos.

En Segundo lugar, que dicha temática pase a formar parte de las políticas nacionales o regionales en lo que se refiere a planificación de asentamientos urbanos y ordenamiento territorial.

En Tercer lugar, focalizar en el fortalecimiento de la organización local para enfrentar los desastres y las emergencias, a través de comisiones locales, campañas de educación y de información pública, reforzando la capacidad individual, familiar y comunal, para enfrentar las amenazas y reducir el riesgo.

Por último, sugerimos la realización de reuniones con políticos, planificadores urbanos, funcionarios municipales, técnicos y organizaciones de manejo de emergencias para discutir e intentar prevenir la situación, o al menos atacarla desde sus orígenes.

Además, es de interés destacar que, cuando la prevención no es suficiente es preciso mitigar los desastres naturales, a partir de la implementación de Planes. Estos planes intentan incorporar no solo los aspectos que hacen a la prevención sino también, a la mitigación de los desastres, a partir de diversas estrategias y líneas de acción, las cuales se enuncian a continuación.

Publicado en formato digital: Mgter. Viviana Claudia Pertile. EL FENÓMENO URBANO Y LA SITUACIÓN EN LOS PAÍSES LATINOAMERICANOS. EMPLAZAMIENTOS URBANOS EN ZONAS DE RIESGOS NATURALES. EL CASO DEL GRAN RESISTENCIA.). Revista Geográfica Digital. IGUNNE. Facultad de Humanidades. UNNE. Año $9 . N^{\circ} 18$. Julio - Diciembre 2012. ISSN 1668-5180 Resistencia, Chaco. En: http://hum.unne.edu.ar/revistas/geoweb/default.htm 
Revista Geográfica Digital. IGUNNE. Facultad de Humanidades. UNNE. Año 9. № 18. Julio - Diciembre 2012. ISSN 1668-5180 Resistencia, Chaco

Estrategia de seguridad y orden, para lo cual se prevén las siguientes líneas de acción:

$>$ Prevenir y atender las emergencias causadas por fenómenos naturales, o de origen humano; apoyando a los organismos que forman parte de la Comisión local de emergencias con el objeto de que adelanten proyectos de investigación, atención y disminución de las vulnerabilidades y riesgos en la ciudad.

> Capacitar a la comunidad en general en temas de atención de desastres por inundaciones, amenazas y riesgos, de acuerdo a la disponibilidad del presupuesto.

$>$ Realizar campañas de divulgación sobre fenómenos naturales y prevención de desastres a nivel escolar, a través de proyectos de educación ambiental.

> Identificar y preservar áreas libres ubicadas en lugares apropiados para que sirvan como refugios temporales en caso de desastre.

En lo que respecta a las estrategias referidas al ambiente, las líneas de acción que persigue el Proyecto de Desarrollo, deberían estar relacionadas de una u otra forma con la Prevención, donde el concepto de Desarrollo debe apoyarse sobre las bases de la Conservación, la cual debe comprender un uso racional del medio ambiente, con el fin de conseguir la más alta calidad de vida para el hombre..., con el Desarrollo se postula una visión de largo plazo, plenamente solidaria con la humanidad toda. Propende a la interdisciplinariedad en la medida que integra una nueva visión del ambiente como fuente potencial de recursos, con sensibilidad hacia los rasgos culturales propios de cada sociedad (Molano Barrero, 1989).

Finalmente en lo que hace a la planificación y al reordenamiento urbano sería interesante considerar algunas líneas de acción, en el marco de la sustentabilidad de proyectos y programas de asentamientos urbanos, entre las cuales podríamos enuncian a continuación:

$>$ Reubicación de asentamientos humanos en zonas adecuadas y de conformidad con los estudios técnicos y universitarios actualizados en la materia.

$>$ Realizar una zonificación a escala micro del área inundable, para que sirva de base determinante para la ocupación del suelo, con la cooperación incluso de organismos internacionales.

$>$ Planificar las Áreas de Expansión Urbana correspondientes al Sur y Oeste, de acuerdo con las necesidades de la ciudad.

> Garantizar el acceso a servicios esenciales y a infraestructura, donde la empresa privada sea la proveedora y el Estado el que garantice la calidad y el acceso a todas las personas.

$>$ Entre otras.

Para llegar a un desarrollo urbano sustentable debemos abordar una dimensión más abarcativa. En efecto, si la noción setentista de ecodesarrollo suponía un matiz alternativista, esto es, una voluntad un tanto externa y algo utópica o romántica, de moderar o equilibrar los excesos que el desarrollo económico infringía a la naturaleza, el concepto de desarrollo sustentable parece constituir una reflexión interna que, si bien no deja de esgrimir argumentos críticos, procura analizar precisamente, la condición, cada vez más delicada, de viabilidad del presente modo productivo. Es decir, una especie de discurso un tanto adaptativo o realista, que en definitiva alienta una cierta imagen de calidad de vida urbana mínima, basada en la posibilidad de una reorganización del modo productivo tal, que asegure tal estándares elementales (Fernández, R. 1998)

\section{Conclusión}

La ciudad es una de las grandes creaciones humanas; la formidable expansión de este proceso de crecimiento explosivo, de desarrollo espacial y de concentración de población permite que este espacio, adquiera la denominación de Fenómeno Urbano.

Este crecimiento inédito de las ciudades trae aparejado dramáticos déficit fundamentales e inclusive altos niveles de criminalidad. Por ello es preciso debatir sobre la seguridad y salubridad de las

Publicado en formato digital: Mgter. Viviana Claudia Pertile. EL FENÓMENO URBANO Y LA SITUACIÓN EN LOS PAÍSES LATINOAMERICANOS. EMPLAZAMIENTOS URBANOS EN ZONAS DE RIESGOS NATURALES. EL CASO DEL GRAN RESISTENCIA.). Revista Geográfica Digital. IGUNNE. Facultad de Humanidades. UNNE. Año $9 . N^{\circ} 18$. Julio - Diciembre 2012. ISSN 1668-5180 Resistencia, Chaco. En: http://hum.unne.edu.ar/revistas/geoweb/default.htm 
ciudades, dentro del criterio clave del "desarrollo sustentable, es decir dentro de los parámetros ambientales.

Las zonas sobtropicales, en la que se encuentra inserto nuestro espacio urbano -objeto de estudio-, poseen la característica de brindar una alta variabilidad, en la oferta climática de precipitaciones. Ello determina momentos de excesos y de déficit hídricos con los que se debe convivir; por lo tanto los modos de vida y los sistemas productivos se deben adaptar a estas condiciones que impone la naturaleza. De no ser así, se originan situaciones de catástrofes y de emergencia -bien conocidas y a las que nos hemos acostumbrado-. La vulnerabilidad esta dada por la combinación de factores que determinan cierto grado de riesgo [2].para un grupo poblacional, provocado por un evento específico y recurrente: en este caso particular son las inundaciones. El riesgo afrontado por la gente tiene que considerarse como una combinación compleja de vulnerabilidad y amenaza o peligro.

Es necesario terminar con la incertidumbre y obligar a las autoridades a orientar las acciones tendientes a prevenir estas situaciones de emergencias traumáticas que la sociedad, en continuo crecimiento, viene soportando desde hace décadas.

Se ha puesto en evidencia que tampoco fue suficiente la existencia de leyes y normas para que exista una solución definitiva. Es entonces necesario crear otro campo de soluciones que incluya el manejo integral del sistema natural, el sistema socio-productivo y el sistema de manejo del poder, dentro del marco de un Sistema Complejo, donde la estructura de la totalidad no solo se puede conocer analizando las relaciones entre sus partes, sino conociendo las reglas de transformaciones que ocurren dentro del sistema; y de esta manera tomarlas medidas pertinentes para que en un futuro próximo, la ciudad sustentable se convierta en una realidad.

\section{Notas}

[1] Durante los primeros estadios de la Humanidad, cuando el Hombre se sentía temeroso ante lo desconocido, solo le quedaba el recurso de transformar aquello en sagrado: dioses buenos o malos. Según pasaron los años, comenzó a comprender a estos fenómenos naturales y mediante la ciencia y la técnica, se libera del miedo a las fuerzas de la naturaleza y comienza a usarla en su favor. Entre otras tantas cosas, pudo manejar el agua. Grandes civilizaciones de la antigüedad llegaron a serlo, por haber crecido como civilizaciones hidráulicas. Las culturas mayas, aztecas e incas, son grandes exponentes americanos de estas civilizaciones.

[2] Según Cardona, Omar Darío. "Manejo Ambiental y Prevención de desastres: Dos temas asociados". en: Ciudades en riesgo. Degradación ambiental, riesgos urbanos y desastres en América Latina. Ediciones LA RED, Lima, Perú. 1996.(Pag.101) Riesgo es la probabilidad de exceder un valor específico de consecuencias económicas, sociales o ambientales en un sitio particular y durante un tiempo de exposición determinado. Se obtiene de relacionar la amenaza, o probabilidad de ocurrencia de un fenómeno, con una intensidad específica, con la vulnerabilidad de los elementos expuestos.

\section{Bibliografía}

Beaujeu Garnier, J.Y Chabot, Georges, 1975: Tratado de Geografía Urbana. Ed. Vicens Vives, Barcelona.

Derruau, Max,1967:Tratado de Geografía Humana. Ed. V. Vives. Barcelona.

Fernández, Roberto, 1998: La Ciudad verde. Manual de gestión ambiental urbana.. Editorial del Centro de Investigaciones Ambientales. Fac. de Arquitectura y Urbanismo. Mar del Plata. Buenos Aires.

Diario Clarín, 1996: Conferencia Mundial sobre asentamientos Humanos, domingo 9 de junio de 1996.

Garcia, Rolando : Conceptos básicos para el estudio de sistemas complejos, Ed. Siglo XXI, México.

Lezama, Jose Luis, 1993: Teoría Social. Espacio y Ciudad. El Colegio de México. México

Maskrey, Andrew, 1993: Vulnerabilidad y Mitigaciones de Desastres. Colombia.

Molano Barrero, Joaquin 1989: Lineamientos teóricos sobre el análisis del paisaje, En COLOMBIA GEOGRÁFICA, Vol..XV- No 1 . Instituto Geográfico A. Codazzi. Bogota, Colombia.

Publicado en formato digital: Mgter. Viviana Claudia Pertile. EL FENÓMENO URBANO Y LA SITUACIÓN EN LOS PAÍSES LATINOAMERICANOS. EMPLAZAMIENTOS URBANOS EN ZONAS DE RIESGOS NATURALES. EL CASO DEL GRAN RESISTENCIA.). Revista Geográfica Digital. IGUNNE. Facultad de Humanidades. UNNE. Año $9 . N^{\circ} 18$. Julio - Diciembre 2012. ISSN 1668-5180 Resistencia, Chaco. En: http://hum.unne.edu.ar/revistas/geoweb/default.htm 
Popolizio, Eliseo,1989: Algunos elementos geomorfológicos condicionantes de la organización espacial y las actividades del NEA, Geociencias $n^{\circ}$ XVII, Centro de Geociencias Aplicadas, U.N.N.E., Resistencia.

Rica, Agustín H. 1990: Hacia un mundo de ciudades. El proceso de urbanización. Cuadernos de Estudio 12. Serie Geográfica. Ed. Cincel. Madrid.

Tyller miller, g., jr. 1992: Ecología y Medio Ambiente: Introducción a la Ciencia Ambiental, el Desarrollo Sustentable y la Conciencia de Conservación del Planeta Tierra. Ed. Grupo Editorial Iberoamérica. México .

Veyret, Ivette yPech, Pierre, (1995): El Hombre y el Medio Ambiente. Ed. Docencia. Bs.As.

Zamorano, Mariano 1977: Geografía urbana. Formas económicas y dinámicas de las ciudades. Colección Geográfica № 6. Buenos Aires.

Publicado en formato digital: Mgter. Viviana Claudia Pertile. EL FENÓMENO URBANO Y LA SITUACIÓN EN LOS PAÍSES LATINOAMERICANOS. EMPLAZAMIENTOS URBANOS EN ZONAS DE RIESGOS NATURALES. EL CASO DEL GRAN RESISTENCIA.). Revista Geográfica Digital. IGUNNE. Facultad de Humanidades. UNNE. Año 9. № 18. Julio - Diciembre 2012. ISSN 1668-5180 Resistencia, Chaco. En: http://hum.unne.edu.ar/revistas/geoweb/default.htm 